\title{
A Review on Back-Propagation Neural Networks in the Application of Remote Sensing Image Classification
}

\author{
Alaeldin Suliman and Yun Zhang \\ Department of Geodesy and Geomatics Engineering, University of New Brunswick, Fredericton E3B-5A3, Canada
}

Received: November 20, 2014 / Accepted: December 20, 2014 / Published: January 25, 2015.

\begin{abstract}
ANNs (Artificial neural networks) are used extensively in remote sensing image processing. It has been proven that BPNNs (back-propagation neural networks) have high attainable classification accuracy. However, there is a noticeable variation in the achieved accuracies due to different network designs and implementations. Hence, researchers usually need to conduct several experimental trials before they can finalize the network design. This is a time consuming process which significantly reduces the effectiveness of using BPNNs and the final design may still not be optimal. Therefore, there is a need to see whether there are some common guidelines for effective design and implementation of BPNNs. With this aim in mind, this paper attempts to find and summarize the common guidelines suggested by different authors through literature review and discussion of the findings. To provide readers with background and contextual information, some ANN fundamentals are also introduced.
\end{abstract}

Key words: Artificial neural networks, back propagation, classification, remote sensing.

\section{Introduction}

One of the most common and important applications in the field of remote sensing is digital image classification [1]. The purpose of image classification is to extract distinct classes of land cover categories from remotely sensed digital images [2]. Recently, artificial intelligence technologies have been involved widely in the automation of remote sensing applications. ANN (Artificial neural network) technology is applied extensively in the classification process of remotely sensed image data. This is due to the ability of ANNs to solve discontinuous and non-linear classification problems, in addition to their robustness, adaptability, and high accuracy [3]. Different kinds of ANNs are constructed with different network architectures; thus different learning algorithms are required [4].

The most common type of ANN used in classification of remote sensing imagery is the MLP

Corresponding author: Alaeldin Suliman, Ph.D. candidate, research fields: photogremmetry, remote sensing, object-based image analysis, and 3D modeling. E-mail: A.suliman@unb.ca. (multi-layer perceptron) networks based on the BP (back-propagation) learning algorithm [1]. This type of network is called BPNN (back propagation neural network). Studies on the performance of back propagation networks are still ongoing. In 2009, Alsmadi et al. [5] concluded that the back propagation learning algorithm is the best algorithm among the Multi-layer perceptron algorithms.

The processing speed and classification accuracy often depend on the design and implementation of the network. In the literature, different researchers introduced different network architectures for different applications. Are there any common standards among neural network researchers to guide the design and implementation of BPNNs for remote sensing image classification? This question is still open. To find some answers, this paper attempts to review and summarize the common guidelines suggested by different authors on the design and implementation of BPNNs. To provide readers with background and contextual information, some ANN fundamentals are also introduced. 


\section{Background and Fundamentals}

The starting point of ANNs development was the invention of the perceptron which was introduced by Rosenblatt in 1958. The perceptron element is a single processing unit that receives weighted input and produces a rule based thresholded output. Linearly separable data can be classified by an SLP (single layer perceptron) whereas non-linearly separable data cannot [1]. MLP is a feed-forward ANN that is developed through a modification of the standard linear perceptron. This modification is made by the use of three or more layers of neurons with nonlinear activation function $[6,7]$. As a result, non-linearly separable data can be classified by MLP unlike the typical perceptron. Fundamentals of ANNs along with their architecture and learning methods are presented and discussed below.

\subsection{ANNs (Artificial Neural Networks)}

Nicoletti [4] defines the ANNs as computational networks consisting mainly of massively parallel processing units that interact with each other through weighted interconnections [4]. This system tries to mimic, in gross manner, the networks of the nerve cells of the human biological central nervous system [7]. The power of these networks depends on the number of these units, their interconnections, and how each unit operates [6].

These processing units are referred to as neurons and each of them receives many input signals, from those to which it is connected, and produces one output which is modulated by an input-output function. A typical neuron model (the standard perceptron) is illustrated in Fig. 1 [8 ]. The influence strength between neurons is determined via numerical weights associated with each interconnection [9].

The network implementation cycle starts by presenting the input data vector to the input neurons. The received input signals at each neuron are processed through a weighted sum and then transmitted as an output to other neurons by a transfer/activation function.

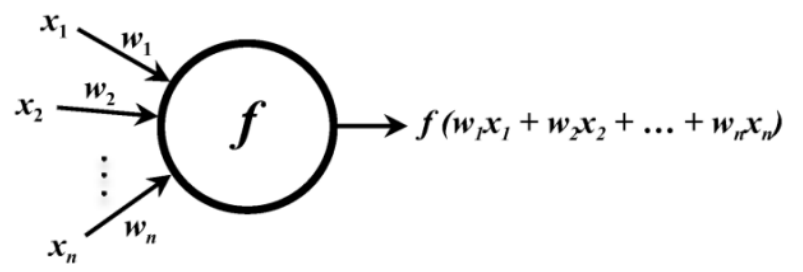

Fig. 1 A typical artificial neuron model [8].

The outputs are scaled through the connection weights and received by the following neurons as input signals. This process will continue in the same manner from one layer to another until the actual output signals are produced at the last layer which contains the output neurons [9].

ANNs should be trained by examples before they can be used. The training process aims to adjust the connection weights and neurons' thresholds by using training data sets. For instance, in a case of supervised learning, the adjustment stage is repeatedly comparing the output for each input pattern with the desired output and propagating the error backward to tune the parameters each time until the error is minimized [9].

The execution stage of the trained networks will be implemented by fixing the connection weights and thresholds which are calculated during the training stage. The network will directly compute the outputs that corresponded to the presented input data. Hence, the calculation time will be short because the network processes the inputs only once [9].

\subsection{ANNs Architecture}

The performance of neural networks is affected directly by the network architecture; thus, it is an important issue. The network architecture includes the connection pattern, number of layers, number of the neurons in each layer, and their activation function [5].

Nicoletti [4] stated that the neural connection patterns fall into two types, the feed-forward and recurrent (feedback) connections. In feed-forward networks the connections are unidirectional; therefore, no loops are presented. Conversely, the recurrent networks contain connection loops due to the 
feedback connections. Therefore, the connections are bidirectional [4]. The multi-layer perceptron network is a well-known example of a feed-forward network. Whereas, Kohonon's neural network is an example of a recurrent network.

Richards [10] underlined that there are three main types of network layers: input, hidden, and output layers. Fig. 2 shows a typical neural network architecture which is also an MLP model [10]. This model consists of three layers. The input layer neurons perform the function of distributing and scaling, if needed, the inputs to the processing elements of the next layer. The hidden layer neurons process the inputs and send the results to the output layer (usually, one hidden layer will be sufficient). Finally, the output layer neurons provide the class labeling information [10].

The number of neurons in each layer is another important issue. Normally, the number of neurons in the input and output layers does not strongly affect the accuracy of the network output results unlike the number of hidden layer neurons. For classification problems, usually the number of the output neurons is related to the number of classes; however, the number of input layer neurons is normally related to the number of bands of the remote sensing image being classified. In contrast, for the hidden layer, the number of the neurons is not readily decided because there is no fixed formula or adopted guideline to determine this number [3].

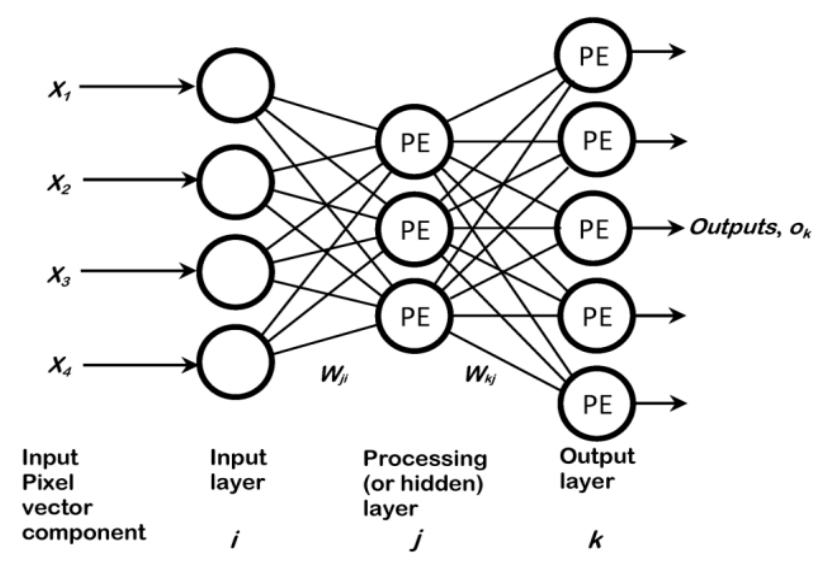

Fig. 2 MLP [10].
Activation function identifies the neuron output in terms of the linear summation of its inputs. This function is also referred to as transfer or input-output function. Basically, these kinds of activation functions are the threshold function, the piecewise-linear function, and the logistic (sigmoid) function [11].

Richards [10] specified that the function's specification is to provide thresholding in a soft or asymptotic sense and be differentiable. This means that the generalized form of a neuron input linear combination will be other than the threshold or linear piecewise functions. The most common type used in classification is the sigmoid activation function because it allows greater similarity to the real biological neurons than do linear or threshold functions. However, all three types must be considered as rough approximations [10]. The common sigmoid function can be expressed as:

$$
f(z)=\frac{1}{1+e^{-z / \theta_{0}}}, z=\sum w x+\tau
$$

where, $z$ is the linear combination of neuron weighted inputs; $\tau$ is a threshold (i.e., bias) that may be included as a weight $\left(w_{0}\right)$ with unity input; $\theta_{0}$ is the steepness constant that governs the function output gradient. However, other functions are preferred in the classification application, which are mainly modified versions of Eq. (1), such as hyperbolic functions $\left(\tanh \left(Z / 2 \theta_{0}\right)\right)$; the mathematical expression is as follows [8]:

$$
f(z)=\frac{2}{1+e^{-z / \theta_{0}}}-1=\frac{1-e^{-z / \theta_{0}}}{1+e^{-z / \theta_{0}}}
$$

Fig. 3 shows a graphical representation of these two common activation functions [11]. The outputs of these functions are illustrated using different values of steepness constant. It is clear that the second function is a rescaled version of the first one. In other words, while the output range for the sigmoid function is unipolar $(0,1)$, the output range for the hyperbolic function is bipolar $(-1,1)$. 


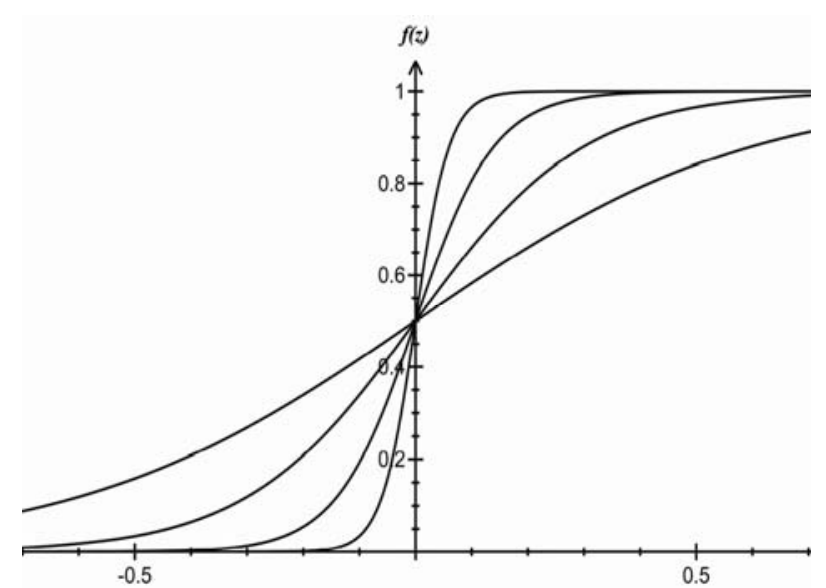

(a)

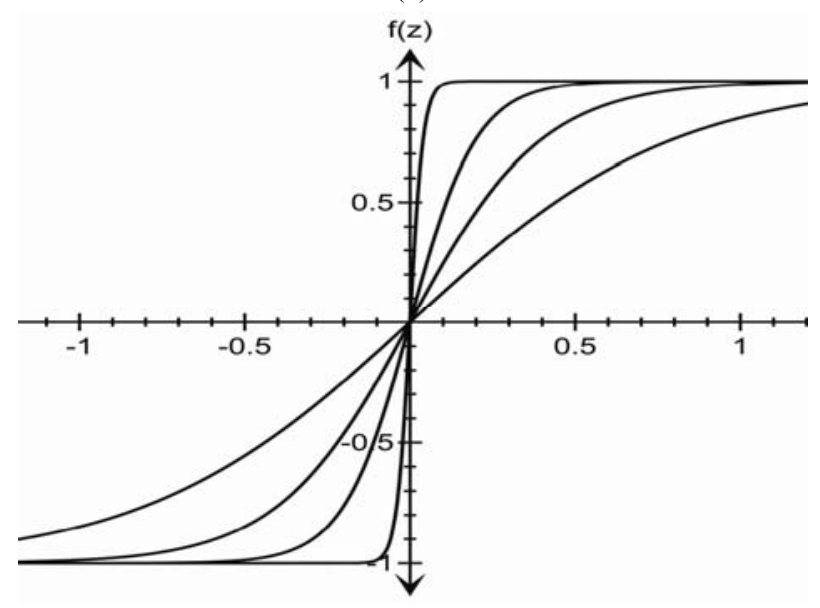

(b)

Fig. 3 Commonly used activation functions: (a) Logistic (Sigmoid); (b) hyperbolic [11].

\subsection{ANNs Learning Methods}

Nicoletti [4] states that the learning process in ANNs can be defined as an adjustment process for the weights of the network connections via examples among the training dataset in order to be able to perform a particular task efficiently. This process will keep on until the error function outputs are minimized. This ability to learn from examples is one of the major advantages of such networks over conventional expert systems [4].

The design of the learning process is constructed according to the type of information available for the network (referred to as learning paradigms) and the rules that govern the updating process (termed as learning rules) [4].
Learning paradigms fall into three categories: supervised, unsupervised, and hybrid. However, the learning rules are of four types: error correction, Boltzmann, Hebbian, and competitive learning. The procedure of implementing the learning rules is referred to as a learning algorithm [4].

Jian et al. [12] summarized the various types of paradigms with their related learning rules, architecture, algorithms, and corresponding applications in a tabular form. Table 1 below illustrates this summary [12].

According to this table, three different learning algorithms have been developed to train the MLP networks: back propagation, delta rule, and perceptron learning algorithm. However, a comparative study done by Alsmadi, Omar, and Noah [5] showed that the BP learning algorithm is the best of the MLP algorithms to train MLP networks.

\section{BP (Back Propagation)}

The BP learning algorithm was first proposed by Paul Werbos in the 1970s. However, it was rediscovered in 1986 by Rumelhart and McClelland, and after that BP became extensively used [5].

The theory of the BP algorithm is based on the error-correction learning rule which uses the error function in order to modify the connection weights to gradually reduce the error. To simplify, this error is the difference between actual network output and the desired output. Therefore, the BP learning algorithm is attached to the supervised learning paradigms because it requires a desired output pattern [7,8].

Eq. (3) shows the expression of the error at the $j t h$ layer; however, for normalization purposes, the error function illustrated in Eq. (4) is used in the derivations of this algorithm.

$$
\begin{gathered}
e_{j}=\left(t_{j}-o_{j}\right) \\
E=\frac{1}{2} \sum_{j} e_{j}{ }^{2}=\frac{1}{2} \sum_{j}\left(t_{j}-o_{j}\right)^{2}
\end{gathered}
$$


Table 1 The well-known learning algorithms of ANNs.

\begin{tabular}{|c|c|c|c|c|}
\hline Paradigm & Learning rule & Architecture & Learning algorithm & Task \\
\hline \multirow{5}{*}{ Supervised } & Error-correction & $\begin{array}{l}\text { Single or multilayer } \\
\text { perceptron }\end{array}$ & $\begin{array}{l}\text { Perceptron learning } \\
\text { algorithms } \\
\text { Back-propagation } \\
\text { Delta rule }\end{array}$ & $\begin{array}{l}\text { Pattern classification } \\
\text { Function approximation } \\
\text { Prediction control }\end{array}$ \\
\hline & Boltzmann & Recurrent & $\begin{array}{l}\text { Boltzmann learning } \\
\text { algorithm }\end{array}$ & Pattern classification \\
\hline & Hebbian & Multilayer feed forward & $\begin{array}{l}\text { Linear discriminant } \\
\text { analysis }\end{array}$ & Data analysis pattern classification \\
\hline & \multirow{2}{*}{ Competitive } & Competitive & $\begin{array}{l}\text { Learning vector } \\
\text { quantization }\end{array}$ & $\begin{array}{l}\text { Within-class categorization } \\
\text { Data compression }\end{array}$ \\
\hline & & ART network & ART Map & $\begin{array}{l}\text { Pattern classification } \\
\text { Within-class categorization }\end{array}$ \\
\hline \multirow{6}{*}{ Unsupervised } & Error-correction & Multilayer feed forward & Sammon's projection & Data analysis \\
\hline & \multirow{2}{*}{ Hebbian } & $\begin{array}{l}\text { Feed- forward or } \\
\text { competitive }\end{array}$ & $\begin{array}{l}\text { Principal component } \\
\text { analysis }\end{array}$ & $\begin{array}{l}\text { Data analysis } \\
\text { Data compression }\end{array}$ \\
\hline & & Hopfield Network & $\begin{array}{l}\text { Associative memory } \\
\text { learning }\end{array}$ & Associative memory \\
\hline & \multirow{3}{*}{ Competitive } & Competitive & Vector quantization & Categorization Data compression \\
\hline & & Kohonen's SOM & Kohonen's SOM & Categorization Data analysis \\
\hline & & ART networks & ART1,ART2 & Categorization \\
\hline Hybrid & $\begin{array}{l}\text { Error-correction } \\
\text { and competitive }\end{array}$ & RBF network & RBF learning algorithm & $\begin{array}{l}\text { Pattern classification } \\
\text { Function approximation } \\
\text { Prediction, control }\end{array}$ \\
\hline
\end{tabular}

where, $j$ denotes the layer index, $t_{j}$ is the desired output, and $o_{j}$ is the actual network output.

The gradient descent technique is a common approach to minimize the value of a function. This principle is utilized in the BP algorithm to adjust the connection weights in order to minimize the error function.

The adjustment process is conducted by modifying the weights via values proportional to the negative of the partial derivative of the error function. This can be expressed as follows [7, 10]:

$$
\Delta w_{j i}=-\eta \frac{\partial E}{\partial w_{j i}}, w_{j i}^{\prime}=w_{j i}+\Delta w_{j i}
$$

where, $\Delta w_{j i}$ is the weight vector updates for the network connections between the layers indexed by $i$ and $j ; \eta$ is a constant referred to as the learning rate; $w_{j i}$ and $w_{j i}^{\prime}$ are the current and updated connection weights respectively for the connections between layers $i$ and $j$.

Basically, the layer $j$ might be the output layer or a hidden layer in the network. Therefore, two cases can be considered. Fig. 2 illustrates the network diagram under consideration and includes the nomenclature used for the following partial derivative equations. These partial derivatives are expanded using the chain rule for simplification $[7,10]$.

First, in the case of the output layer (layer $k$ ):

$$
\begin{aligned}
\frac{\partial E}{\partial w_{k j}} & =\left(\frac{\partial E}{\partial o_{k}} \cdot \frac{\partial o_{k}}{\partial z_{k}}\right) \cdot \frac{\partial z_{k}}{\partial w_{k j}}=\frac{\partial E}{\partial z_{k}} \cdot \frac{\partial z_{k}}{\partial w_{k j}} \\
& =\left(-e_{k} \cdot f^{\prime}\left(z_{k}\right)\right) \cdot o_{j}=-\delta_{k} \cdot o_{j}
\end{aligned}
$$

Second, in the case of the hidden layer (layer $j$ ):

$$
\begin{aligned}
& \frac{\partial E}{\partial w_{j i}}=\left(\quad\left[\frac{\partial E}{\partial o_{j}}\right] \cdot \quad \frac{\partial o_{j}}{\partial z_{j}}\right) \cdot \frac{\partial z_{j}}{\partial w_{j i}}=\frac{\partial E}{\partial z_{j}} \cdot \frac{\partial z_{j}}{\partial w_{j i}} \\
& =\left(\left[\sum_{k} \frac{\partial E}{\partial z_{k}} \cdot \frac{\partial z_{k}}{\partial o_{j}}\right] \cdot f^{\prime}\left(z_{j}\right)\right) . o_{i} \\
& =\left(\sum_{k}\left[\left(-e_{k} \cdot f^{\prime}\left(z_{k}\right)\right) \cdot w_{k j}\right] \cdot f^{\prime}\left(z_{j}\right)\right) \cdot o_{i}=-e_{j} \cdot f^{\prime}\left(z_{j}\right) \cdot o_{i} \\
& =-\left(\sum_{k}\left[\delta_{k} \cdot w_{k j}\right] \cdot f^{\prime}\left(z_{j}\right)\right) \cdot o_{i} \quad=-\delta_{j} \cdot o_{i}
\end{aligned}
$$

where, $e$ is the difference between the desired and the actual output "i.e., the error" at the layer denoted by the subscript; $o$ refers to the weighted output of the layer denoted by the subscript; $f^{\prime}$ (.) refers to the first derivative of the activation function; $z$ is the activation 
input which is the linear summation of the weighted inputs for each neuron at the layer denoted by the subscript; and $\delta$ is the local gradient of the error energy function with respect to the activation input $z$ at the layer denoted by the subscript. Thus, referring to Eq. (5) the updated connection weights will be as follows [7, 10]:

For the output layer $(k)$ :

$$
w_{k j}^{\prime}=w_{k j}+\eta \cdot \delta_{k} \cdot o_{j}
$$

For a hidden layer $(j)$ :

$$
w_{j i}^{\prime}=w_{j i}+\eta \delta_{j} \cdot o_{i}
$$

After choosing the weights of the network randomly, the BP algorithm is used to compute the necessary corrections. The algorithm can be decomposed in the following three steps: (a) the computation of a feed-forward stage, (b) backward propagation of the error to both the output layer and the hidden layers, and (c) update of the weights for the network connections. The algorithm is stopped when the value of the error function has become less than a predefined acceptable tolerance.

It is important to mention that the BP algorithm can be implemented in two modes, batch and incremental mode. In batch mode the network weights are updated once after the presentation of the complete training set. However, in incremental mode the weights are updated immediately after each pattern presentation. In general, incremental BP algorithm does not converge to a single point in weight space, but oscillates around the minimum of the error function [13].

After the corrections have been adopted, the testing stage starts to evaluate the attainable accuracy of the trained neural network. This stage will be very fast because the network only needs to calculate the corresponding output to the presented set of input data [8]. The coming subsections discuss the limitations of the BP algorithm and how it can be improved.

\subsection{Limitations of BP Algorithm}

According to Alsmadi et al. [5], BP is considered as the best algorithm for the multilayer perceptron. However, it still has several limitations that may be summarized in the following five points:

(1) Long learning time: the speed of the obtained convergence is very slow.

(2) Selective learning data: BP is a supervised learning algorithm; therefore, numerous input-output training examples are required. Furthermore, the accuracy of the output is affected significantly by the selected training data. Thus, good training data selection is required for high-quality result.

(3) Local minima possibility: the network may achieve an error that is lower than the surrounding possibilities and it is not the smallest possible one. This is called a local minimum which means that the result may generally converge to any local minimum on the error surface, since stochastic gradient descent exists on a surface which is not flat. To clarify this concept, Fig. 4 illustrates an example of a local minimum [8].

(4) Unguaranteed convergence: it is not guaranteed that the network will converge to an acceptable output because of its high possibility of getting stuck in local minima.

(5) Black box: the internal mapping procedures of the BP are still unclear and not realized.

\subsection{Acceleration of BP}

Due to the limitations of the BP learning algorithm, mainly the convergence oscillation and learning time,

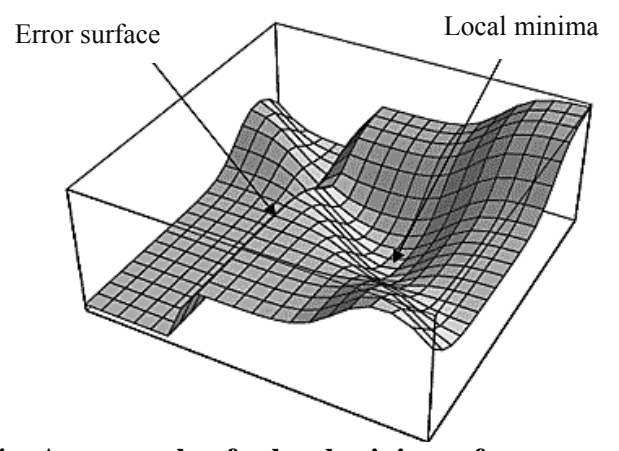

Fig. 4 An example of a local minima of an error function [8]. 
some modifications and improvements have been proposed to accelerate the learning process in neural networks. Two of the common modifications are briefly introduced below:

\subsubsection{BP using Momentum}

This modification tries to orient the gradient of the error function to the center of the error surface by introducing a momentum term. The concept of momentum is that the current movement direction in the weight space is influenced by the previous changes in the weights. This improvement calculates the current weight for a connection then adds a proportional part of the previous calculated weight correction of that connection. Thus, the current weight correction can be expressed as [8]:

$$
\Delta w_{k j}(n)=\eta \cdot \delta_{k} \cdot o_{j}+\alpha \cdot \Delta w_{k j}(n-1)
$$

where, $\eta$ and $\alpha$ are the learning rate and the momentum constant respectively; $\Delta w k j(n)$ and $\Delta w k j(n-1)$ are the weight corrections for the current epoch $(n)$ and previous epoch $(n-1)$ respectively [8].

3.2.2 BP using LM (Levinburg Marquardt) Algorithm

A more stable method is to use the BP learning algorithm based on an algorithm called the Levinburg Marquardt training algorithm (LM algorithm). Generally, like the aim of gradient descent, it provides a numerical solution to the problem of minimizing a nonlinear function over its space parameters [14].

This algorithm is designed to approach second-order training speed without computing a Hessian matrix. The current updated weights can be calculated from the following formula:

$$
w_{k j}(n+1)=w_{k j}(n)-\left[J^{T} J+\mu\right]^{-1} \cdot g
$$

and the gradient $(g)$ can be calculated as:

$$
g=J^{T} e
$$

where, $I$ is an identity matrix; $\mu$ is a gradient constraint; $e$ is the error vector of the network; and $J$ is the Jacobian matrix containing the first derivative error function of the network with respect to the weights and thresholds. This matrix can be determined using the standard BP technique [14].

\section{Design and Implementation of BPNNs (Back Propagation Neural Networks)}

This section introduces the current ways of architecture layout design of BPNNs and the selection of the parameters needed for implementation.

\subsection{Number of Layers and Neurons}

Choosing the number of layers and the number of neurons in each layer is essential. The performance of the feed-forward BPNNs is affected significantly by the network layout. The selection of this layout and its related parameters is referred to as the art of network design. Unfortunately, there is no clear answer for the layout of the neural network for a particular application; however, there are some general rules which have been collected over time and followed by researchers [5].

For the number of layers, Alsmadi et al. [5] emphasized that the typical BPNNs should consist of input, hidden, and output layers. Toshniwal [15] recommended that in the case of a multilayer perceptron at least one hidden layer should be used, and this number should be increased to two or more if the number of classification classes is more than twenty.

Usually, there are different numbers of neurons in each layer. In digital image classification problems, the number of neurons in the input layer is usually equal to the number of bands in the image being classified. However, the number of output neurons commonly equals the number of the required classes to be classified. These two numbers are usually selected according to the designer's arrangement.

Contrarily, the number of neurons in the hidden layer is not readily determined. There are some empirical formulas proposed by researchers, but the best way is to be found experimentally. 


\subsection{Initial Values for Weights}

McAuley [16] emphasizes that the initial values of the weights must be both unequal and small. Equality of the initial weights prevents the network from learning in the cases when the solution to a problem requires that the network learns by unequal weights. He explained that this is due to the proportionality between the back propagated error through the network and the value of the weights. For instance, if all the weights are equal, the back propagated errors will be equal, and consequently all of the weights will be updated equally. Thus, to avoid this equilibrium problem, the initial weights of the network should be unequal [16].

Moreover, the initial weights should be small. This is because the weights in the BP algorithm are updated proportionally to the derivative of the activation function (the sigmoid function). For further clarification, the first derivative of Eq. (1) for a steepness constant equals a unity value, the sigmoid function, is shown below in Eq. (13) along with its graphical representation as it is illustrated in Fig. 5.

$$
\frac{\partial}{\partial z} f(z)=f^{\prime}(z)=f(z) \cdot[1-f(z)]
$$

It is clear, from Fig. 5, that the maximum value of this derivation is when $f(z)$ equals to 0.5 and approaches its minimum (zero) as the output of the activation function $-f(z)$-moves toward 0 or 1 . Thus, weights will be changed mainly for neurons of outputs closest to 0.5 (the steepest portion of the sigmoid, Fig. $3)$. Once a neuron activation output becomes close to 0 or 1 , its associated weight update will change very little. Therefore, selecting small initial weights will produce neuron outputs close to 0.5 which generates the highest weight update [16].

\subsection{Learning Rate and Momentum}

Generally, the higher the learning rate $(\eta)$, the higher the weight changes on each iteration (epoch), and the quicker the network learns. However, the learning rate value may prevent the system from achieving a stable solution. If the learning rate gets too high, oscillation of the weights is often the result.

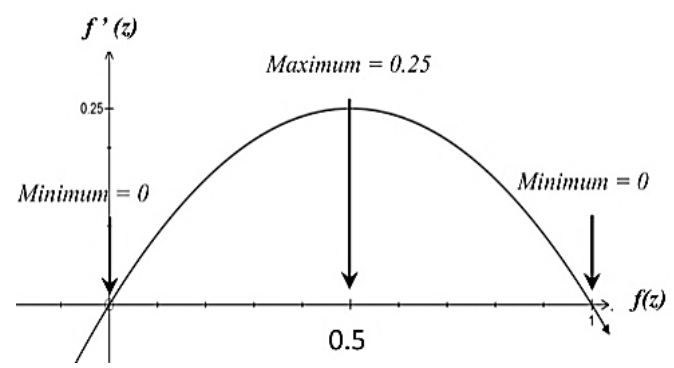

Fig. 5 A graphical representation of the activation function first derivation.

Therefore, the ideal choice is to use the highest learning rate possible without causing oscillation. This choice can be achieved through utilizing a momentum term $(\alpha)$. This term will help the network weights to pass the local minima and speed up the learning process as well.

A value of 0.25 for learning rate and 0.9 for momentum parameter can be started with or applied to a variety of problems when BP learning algorithm is used. However, for difficult tasks, the learning rate might be decreased to 0.01 [16]. An example of such a task is the classification of a high resolution satellite images. Jiefeng et al. [3] used 0.01 and 0.8 for the learning rate and momentum parameters respectively in a classification of high resolution satellite image by BPNN [3]. These values depend on the ANN designs and application; thus, these values are adopted after an investigation stage for the training samples.

\subsection{BP Classification Procedure}

BPNNs are usually used to classify remote sensing images by using a supervised classification procedure. This procedure involves the supervised learning algorithm that is implemented by using a set of input-output training examples.

After designing the network architecture and defining the required classification classes, the training stage starts by presenting the input learning data and adjusting the connection weights and thresholds repeatedly according to the desired output data and predefined classes. The training stage will 
continue until an acceptable error has been achieved. Hence, the network is theoretically ready to be used in a particular classification application and produce a classified image [3]. Fig. 6 shows flowcharts of the two broad steps involved in the classification of the remote sensing images as adopted by Jiefeng et al. [3].

\subsection{Validation and Accuracy Assessment}

To accept or reject the results achieved, always there is a validation stage after the training stage. This stage helps to assess and predict the achievable accuracy of the network for the classified image produced. To test a network's classification performance, the training data are usually divided into two parts. The first part is to train the network and the second is to test and calculate the accuracy measures. The test data are well selected and grouped in classes as they are used in the training stage. The test starts by presenting each class as input data and then recording the actual network outputs in order to construct the so-called confusion matrix [10].

The confusion matrix contains the numbers of pixels that are classified in the network's actual output of the input test data [17]. This matrix is used to compute most of the accuracy measures. Producer accuracy, user accuracy, overall accuracy, and kappa coefficient are still the most widely used measures for accuracy assessment.

\section{Discussions}

A number of relevant scientific resources have been reviewed on the subject of ANNs in general and on the classification application of BPNNs in particular. The sources include books, papers and online articles. It is clearly found that the most common ANN applications in processing remote sensing digital images fall into classification and pattern recognition applications [18], and the most common network architecture used for the classification of remote sensing is the feed-forward BPNN [1]. This is due to the high success of such a network type in performing various image processing applications, especially classification problems in agriculture [19]. The discussion below is going to cover three different aspects of BPNNs: layout, optimization, and accuracy.

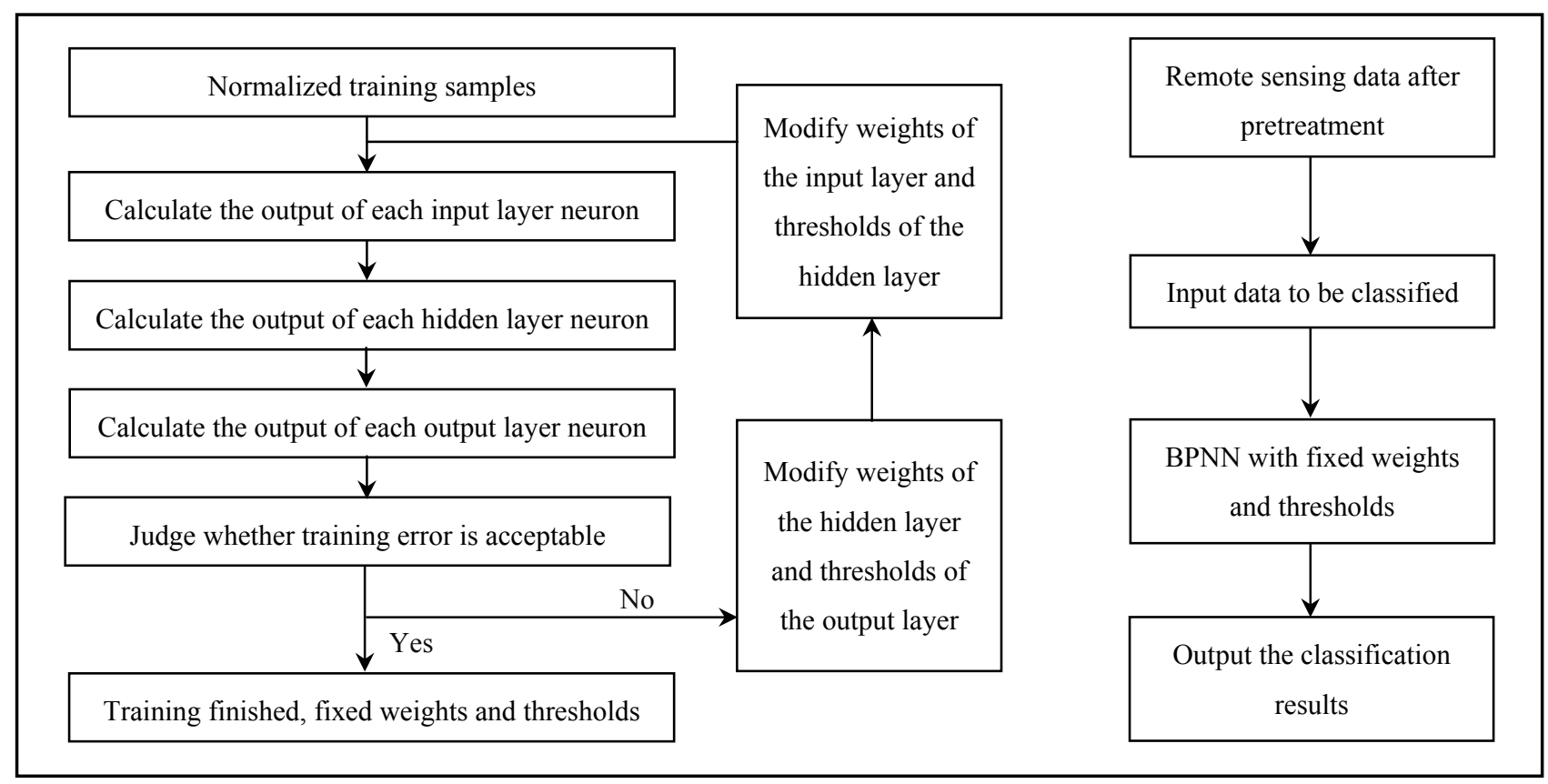

(a)(b)

Fig. 6 The steps of the BP classification procedure: (a) training process to find the final weights and thresholds of BPNN; (b) the classification process of a remote sensing image using the trained BPNN [3]. 


\subsection{Different Layout and Performance}

According to the reviewed material, several classification tasks are investigated through different BPNN architectures. These tasks include utilization of neural networks in classifying digital images such as hand digital camera images, IRS-1D satellite images, sonar images, multi spectral images, SPOT-HRV images, Landsat TM images, and QuickBird high resolution satellite images.

BPNNs have shown great ability to produce classified images with very high accuracies. This can be noticed from the acceptable accuracies achieved in all above mentioned examples. Moreover, in many research papers BPNN was compared with the best statistical classification approach which is MLC (Maximum Likelihood classification) and the results definitely demonstrated the advantages of BPNNs. However, these accuracies still need to be increased and the BP learning algorithm needs to be optimized.

The network layout means mainly the schemas of input, hidden, and output layers. The effects of the network layout are studied in most of the reviewed research papers. These studies include the effect of the output schemes to represent the output data. For instance, Yang et al. [19] tried two output schemes to represent two classes. One output neuron of 0 and 1 coded outputs, and two output neurons of $(1,0)$ and $(0$, 1) coded outputs [19]. In other words, the number of output neurons for the first case is equal to the logarithm of the number of classes $-n$ - to the base of two $-\log _{2}(n)$-; whereas, for the second case, it is equal to the number of classes.

Toshniwal [15] said that the number of the output neurons may be equal to the number of required classes -n- or $\log _{2}(n)$ based on the code followed for the output [15]. This statement infers that the network result will not be affected by the number of output neurons. However, the paper of Yang et al. [19] showed that the results achieved, which were based on the Brier score, by using two output neurons is generally better than using one neuron for the same number of input and hidden neurons [19]. The assessment showed that the difference in the average computed Brier score is about 6\%. Although this difference is small, it evidences that the number of output neurons affects the obtained results, and therefore needs to be further investigated.

For further clarification, Brier score is a score function that evaluates the accuracy of a set of probability assessments by measuring the average squared deviation between predicted probabilities for a set of events and their outcomes [20]. Thus, the lower the Brier scores the higher the network ability in image classification.

Yang et al. [19] presented in their paper tabulated results of the achieved accuracies (i.e., Brier score) by several neuron numbers for a single hidden layer. It would be valuable if these results are graphed and investigated precisely.

The number of neurons in the hidden layer has a great effect on the network performance. Unfortunately, this number is not readily determined. Some empirical equations are proposed to determine the neurons number of the hidden layer. Liang et al. [14] recommended a formula based on their previous experience. The formula used in their research paper is as follows [14]:

$$
n=\sqrt{n_{i}+n_{o}}+a
$$

where, $n$ is the number of the hidden nodes; $n_{i}$ is the number of the input layer; $n_{o}$ is the number of the output layer; $a$ is a constant between 1 and 10 .

According to Toshniwal [15], another formula is proposed to find the number of hidden neurons $\left(N_{h}\right)$. The formula is as follows [15]:

$$
N_{h}=N_{p} \times \sqrt{\left(N_{i}+N_{o}\right)}
$$

where, $N_{p}$ is the number of training samples; $N_{i}$ is the number of input neurons; and $N_{o}$ is the number of output neurons. 
When these formulas are compared to other examples of neural networks in which the number of the hidden neurons was determined by experiments, the first formula is found to be a rough indicator of the number of hidden neurons adopted experimentally; whereas, the latter is found to give an over estimation.

Alsmadi et al. [5] suggested a rule to fix the upper limit of neurons in the hidden layers based on the amount of the available learning data. The calculation of this limit can be defined as follows:

$$
N u_{h}=\frac{N t}{N_{\text {in }} \cdot N_{\text {out }} \cdot s f}
$$

where, $N u_{h}$ is the upper limit number of the hidden neurons; $N t$ is the number of training input-output examples; $N_{\text {in }}$, $N_{\text {out }}$ are the numbers of input and output neurons respectively; and $s f$ is a scale factor between 5 and 10. Larger scale factors are used for relatively noisy data [5].

It is worth mentioning that all of the researchers who propose such formulas, in the reviewed papers, did not follow any of the formulas' results. Therefore, Jiefeng et al. [3] recommended that it is better to determine this number through the trial-and-error method. This makes sense as long as there is no accurate guideline for that.

The number of input neurons in all of the reviewed classification networks is selected to be equal to the number of bands of the image being classified. Furthermore, the reviewed materials did not show any investigations of this number in the architecture design stage. This means that there is no effect of the number of the input neurons on the network results, as long as the aim of these neurons is to distribute and scale the inputs to the processing elements of the next layer. Therefore, it is easier in the design of image classification networks to set the number of input neurons equal to the number of image bands.

\subsection{BP Optimization}

Great improvements can be made on the BP learning algorithm and on the input data. For the BP algorithm, Jiefeng et al. [3] suggested the use of momentum term in the phase of weights update calculations as it is defined in Eq. (10). However Aria et al. [13] and Liang et al.[14] recommended strongly the use of LM algorithm because it can converge rapidly without falling in local minimum. They concluded that it is faster and more accurate than the standard BP algorithm. Aria et al. [13] said that, in a batch mode algorithm, it can converge from ten to one hundred times faster than the standard BP algorithm based on the delta rule. Furthermore, Liang et al. [14] reported that this algorithm helps to avoid the problem of local minima error, speed up the convergence, and prevent oscillating.

By comparing the overall accuracies achieved by using both modifications, it is found that the accuracy reached by using the first modification is higher than that by using the second one by a difference of about $5 \%$ on average. However, this is not a guarantee that the momentum term modification is better than the LM algorithm due to the quality variation and source difference of the images employed. While, the classified image used in the study published by Jiefeng et al. [3] was a QuickBird high resolution satellite image, the image used in the study of Aria et al. [13] and Liang et al. [14] were Landsat TM image and IRS-1D satellite image respectively. This draws a great attention to the input data quality.

The difference in the image resolution gives us an indication of the effect of the input image quality. In other words, the higher the resolution, the better the classification accuracy. That is why many researchers propose different ways to improve the image representation before applying classification. Toshniwal [15] emphasized strongly that the remote sensing image data should be enhanced and corrected both radiometrically and geometrically before it can be used in a classification task.

Training dataset is another important issue. The efficiency of the network performance depends significantly on the training data. Therefore, different 
preparation methods for the training data are introduced to enhance the classification quality. One simple method proposed by Liang et al. [14] is to input the sample data in a matrix and then the minimum and maximum of each column are counted. After that, the modified input data value for each element in the training matrix can be calculated from the following formula:

$$
G(i, j)=\frac{M_{\max }(j)-M(i, j)}{M_{\max }(j)-M_{\min }(j)}
$$

where $G$ is the training sample matrix; and $M$ is the input sample matrix where $i$ and $j$ are the row and column of the image used respectively[14].

A similar optimized approach with a slight difference is recommended by Jiefeng et al. [3]. This approach involves an input data normalization to be between $(0,1)$. The normalization formula can be defined as follows:

$$
G(i, j)=\frac{M(i, j)-M_{\min }}{M_{\max }-M_{\min }}
$$

where, $G$ is the normalized matrix; $M_{\max }$ and $M_{\min }$ are the maximum and the minimum values of the original entire training data; and $M(i, j)$ is the original value of the input vector. By using this data normalization, Jiefeng et al. [3] stated that the saturation in the process of network broadcasting will be avoided.

Another approach is to investigate the data structures' patterns via utilizing the unsupervised learning procedure. This technique allows determining the distinguishable clusters within the data. Such an approach is suggested by Toshniwal [15].

For the case of feature recognition and classification, Sekeroglu [21] proposed a good approach for input data preparation. The approach is used successfully in classification of a sonar image. This method is done by normalizing the input data to be a $100 \times 100$ pixels image in order to find the average pixel per neuron ratio. This average is used as an input data to the BPNN. This pixel/node approach is an efficient approach that makes the training generalization faster.

\subsection{BPNN Accuracy and Assessment}

The most focused accuracy measure in the field of digital image classification is the kappa coefficient because it utilizes most of the confusion matrix parameters. Most of the researchers in this area have used this measure in addition to the overall accuracy as concluding indicators about entire classification accuracy. In general, the accuracy ranges of the reviewed papers are between $63.5 \%$ and $93 \%$ for the overall accuracy and between 0.51 and 0.9 for the kappa coefficient measure. These measures prove that BPNNs have great classification ability and the possibility of higher accuracy achievement by further research. However, this variation in the accuracy is due to several factors, mainly the lack of standards in layout design, input and training data preparation, and implementation parameters selection.

\section{Conclusion}

According to the reviewed papers, it is found that the BPNN is a powerful tool to perform classification tasks for remote sensing images. However, there is no clear and accurate design specification in the area of remote sensing image classification. The BP learning algorithm is used widely to train such networks. It has been proven that it is the best algorithm among the MLP algorithms; however, algorithm optimization and input data preparation are strongly required to be made.

The architecture of the neural networks affects significantly the achieved accuracy, particularly the number of layers and the number of neurons in each layer. It is strongly recommended that the number of layers for classification should be at least three layers (input, hidden, and output layers). This number may be increased if the number of classes to be classified is more than twenty.

Regarding the number of neurons in each layer, it is found that the network accuracy and performance is 
mainly affected by the number of neurons in the hidden layers. However, this effect can be ignored concerning the number of neurons in both input and output layers.

For the layout of image classification networks, researchers commonly design the input and output layers to have a number of neurons equal to the number of the image bands and the number of required classes respectively. They also emphasized that these networks must include at least one hidden layer. However, there is no clear and robust rule to determine the number of neurons in the hidden layers; therefore, almost all of the researchers find this number experimentally after a number of experimental trials.

The quality of the input data is an important issue. Preparation for the input data would ensure better results. These preparations for the input images include mainly radiometric and geometric enhancements and corrections along with data normalization.

To minimize the localization problems and increase the learning speed, a momentum parameter, or other modification, must be used in the BP learning algorithm.

Cross validation calculations must be done after the learning stage to ensure the classification ability and assess the accuracy of the classified image. The variation in the achieved classification accuracy of BPNNs is mainly due to the lack of standards or guidelines in the selection of both architecture and implementation parameters.

\section{Recommendation}

It is strongly recommended that all the BPNN layout and implementation parameters which affect the efficiency and the accuracy of the digital image classification must be tested individually. These tests aim to investigate the results in order to come up with robust guidelines that help researchers in both the network design and implementation stages. Otherwise, the researchers will need to continually conduct several experimental trials before they finalize their network design without guarantee that they adopted the optimal one. Furthermore, the time and effort expended in these experiments will make ANN technology inefficient and unattractive which consequently will prevent its development progress.

Therefore, future research should start by investigating the effects of ANN architecture, namely the number of the neurons in the hidden layers and the network layers as well, on the performance of the BPNNs to construct a design guideline.

\section{Acknowledgements}

This research is supported in part by the Libyan Ministry of Higher Education and the CRC (Canada Research Chair) Program. The authors also greatly appreciate the valuable comments from the anonymous reviewers and the editor for improving this paper.

\section{References}

[1] Atkinson, P., and Tatnall, A. 1997. "Introduction Neural Networks in Remote Sensing." International Journal of Remote Sensing 18 (4): 699-709.

[2] Jindal, S., and Josan, G. 2007. "Neural Network and Fuzzy Logic Approach for Satellite Image Classification: A Review." In Proceedings of COLT, 1-4.

[3] Jiang, J., Zhang, J., Yang, G., Zhang, D., and Zhang, L. 2010. "Application of Back Propagation Neural Network in the Classification of High Resolution Remote Sensing Image: Take Remote Sensing Image of Beijing for Instance." In Proceedings of 18th International Conference on Geoinformatics, IEEE, 1-6.

[4] Nicoletti, G. 2000. "An Analysis of Neural Networks as Simulators and Emulators." Cybernetics and Systems 31 (3): 253-82.

[5] Alsmadi, M., Omar, K., and Noah, S. 2009. "Back Propagation Algorithm: The Best Algorithm among the Multi-layer Perceptron Algorithm," IJCSNS International Journal of Computer Science and Network Security 9 (4): 378-83.

[6] Abu-Mahfouz, I. 2005. "A Comparative Study of Three Artificial Neural Networks for the Detection and Classification of Gear Faults." International Journal of General Systems 34 (3): 261-77.

[7] Graupe, D. 2007. Principles of Artificial Neural Networks, Singapore, Hackensack, N. J.: World Scientific. 

Sensing Image Classification

[8] Rojas, R. 1996. Neural Networks: A Systematic Introduction. Berlin: Springer.

[9] Wang, T. S., Chen, L., Tan, C. H., Yeh, H. C., and Tsai, Y. C. 2009. "BPN for Land Cover Classification by Using Remotely Sensed Data." In Proceedings of Fifth International Conference on Natural Computation, IEEE, 535-9.

[10] Richards, J. 2006, Remote Sensing Digital Image Analysis, Berlin: Springer-Verlag.

[11] Mas, J. F., Mas, and Flores, J. J. 2008. "The Application of Artificial Neural Networks to the Analysis of Remotely Sensed Data." International Journal of Remote Sensing 39 (3): 617-63

[12] Jain, A. K., Mao, J., and Mohiuddin, K. M. 1996 “Artificial Neural Networks: A Tutorial." Computer 29 (3): 31-44.

[13] Aria, E. H., Amini, J., and Saradjian, M. 2003 "Back Propagation Neural Network for Classification of IRS-1D Satellite Images." Joint Workshop of High Resolution Mapping from Space, Tehran University, Iran.

[14] Liang, P., Zhaoyang, X., and Jiguang, D. 2010. "Application of BP Neural Network in Remote Sensing Image Classification." In Proceedings of ICCASM, IEEE, V10-212-V10-215.

[15] Toshniwal, M. “An Optimized Approach to Application of Neural Networks to Classification of Multispectral, Remote Sensing Data." In Proceedings of Networking, Sensing and Control, IEEE, 235-40.

[16] McAuley, D. 2010. "The Back Propagation Network: Learning by Example." School of Information Technology and Electrical Engineering, The University of Qweensland Australia.AccessedAugust24, 2012, http://itee.uq.edu.au/ cogs2010/cmc/chapters/BackProp/.

[17] Lillesand, T. M., and Kiefer, R. W. 1994. Remote Sensing and Image Interpretation, New York: Wiley.

[18] De Ridder, M., and Handels, H. 2002. "Image Processing with Neural Networks-A Review," Pattern Recognition 35 (10): 2279-301.

[19] Yang, C., Prasher, S., Landry, J., and Di Tommaso, A. 2000. "Application of Artificial Neural Networks in Image Recognition and Classification of Crop and Weeds." Canadian Agricultural Engineering 42 (3): $147-52$

[20] Brier, G. W. 1995. "Verification of Forecasts Expressed in Terms of Probability." Monthly Weather Review78 (1): 1-3.

[21] Sekeroglu, B. 2004. "Classification of Sonar Images Using Back Propagation Neural Network.”In Proceedings of IGARSS'04, IEEE International, V5-3092-V5-3095. 